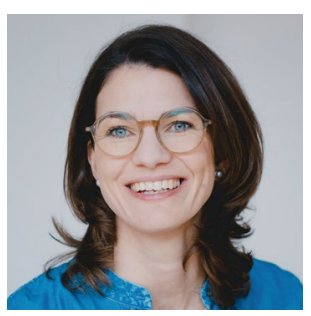

Credit: Marco Borggreve

\title{
How behavioural science data helps mitigate the COVID-19 crisis
}

\author{
In the current absence of medical treatment and vaccination, the unfolding COVID-19 \\ pandemic can only be brought under control by massive and rapid behaviour change. To \\ achieve this we need to systematically monitor and understand how different individuals \\ perceive risk and what prompts them to act upon it, argues Cornelia Betsch.
}

A $\mathrm{t}$ the moment, acting according to official recommendations regarding COVID-19 may feel unreasonable or unnecessary. This may especially be the case in countries where avoiding physical contact with other people is still voluntary and hasn't (yet) been turned into strict policies of closed schools, closed playgrounds or closed shopping centres. Avoiding handshakes with colleagues and students, not hugging friends, turning down a friend's invitation for dinner-you very easily start to wonder whether you are overreacting or whether it is just the right thing to do.

The tragedy of public health preventive measures such as physical distancing is that we do not see and feel when we do not infect someone, when we are part of something not happening, such as transmission chains that can be deadly for our loved ones. We all know someone vulnerable whom we do not want to lose. Not seeing them now, avoiding physical contact, seems like the opposite of a declaration of love, and may make us hesitate to act.

Still, in this pandemic, fast and massive behavioural change is key. Pharmaceutical measures such as a vaccine or antiviral medicines will not be available for COVID-19 for a long time. We have long recognised that understanding behaviour is the basis of changing it: learning about people's behaviour in this crisis is vital. This must include insight into public perceptions of risk, protective and preparedness behaviours, public trust, knowledge and misinformation. Although we have some idea about people's behaviour in public health crises from previous pandemics, a lot has changed: the virus, the ways people gather information and the ways authorities such as WHO reach out to people via social media. Assessing these variables is of great relevance.

To address the pressing need for reliable, ongoing information on the German public's response to COVID-19, we set up the COVID-19 Snapshot Monitoring (COSMO) initiative (https://doi.org/10.23668/ psycharchives.2776). Each week, 1,000 participants-representative for age, gender and federal state-take part in a 15 min online survey. Our goal is to enable the government, journalists and health organizations to be aware of the psychological situation, implement adequate responses, correct misinformation and also facilitate behavioural change, whether with communication measures, policies or restrictions.

This initiative aims to offer a rapid evaluation tool of what the public thinks and feels, including which fears are relevant, the prevalence of hoarding behaviour, discrimination and stigma, trust in information sources and trust in the government. We publish a weekly update for project partners, government officials and journalists registered with Science Media Centre Germany.

In the first two waves of the survey (3-4 March 2020 and 10-11 March 2020), we found that, although knowledge was high, important protection behaviours were very low, and risk perceptions were especially low among the elderly. Authorities can act upon that knowledge to protect this susceptible yet still complacent group. We also found that willingness to restrict one's everyday life, to flatten the curve and lower the burden for the health system, was high. However, when the motivation was to protect vulnerable others, the willingness to restrict one's everyday life was even higher. This is a very important message. Communicating the social norm is a key strategy in health communication. Such data can improve the outbreak response: knowing that the clear majority of people are restricting themselves to protect others takes away the burdening question of "Am I the only fool who does this?" It can create much-needed solidarity at a time when all may suffer from the non-health-related side effects of the crisis.

On the forefront of bringing behavioural insights to the heart of national pandemic response, the WHO Regional Office for Europe has adapted the COSMO study protocol and questionnaire, and now offers support to its Member States to collect such data (http://www.euro.who.int/en/covid19-BI; protocol and materials at https://doi. org/10.23668/psycharchives.2782).

Identifying groups with low risk perceptions and gathering data for social norm communication are only two examples of the power of these data. Scientists across disciplines should continue to collaborate to create a standard set of questions to assess risk perceptions, drivers of protective behaviours and trust, consistently across countries, with necessary cultural and other adaptations as needed. Understanding how we feel and think about the risk we face and how it relates to psychological and societal consequences requires insights from numerous fields. Thus, cross-disciplinary collaboration on such large-scale surveys will be important. The list of important questions to address is long (and will grow as the crisis unfolds): resilience, coping with stressful events, solidarity, the role of religion, loneliness, domestic violence, prosocial behaviour and its driving factors, intergroup conflicts, misinformation, shifts in political opinions and movements, and the role of language in crisis response, to name just a few.

If avoiding people even though neither you nor any of them is actually sick feels stupid, know that you have done the right thing: you limited unnecessary physical contact. Knowing that other people do the same can hopefully lead to more behavioural change and more willingness to act upon the knowledge that we have. Data gives authorities and journalists a solid base for supporting the public to help mitigate the crisis.

\section{Cornelia Betsch ${ }^{1,2}$ \\ ${ }^{1}$ Center for Empirical Research in Economics and Behavioural Sciences (CEREB), University of Erfurt, Erfurt, Germany. ${ }^{2}$ Media and Communication Science, University of Erfurt, Erfurt, Germany. e-mail:cornelia.betsch@uni-erfurt.de}

Published online: 27 March 2020 https://doi.org/10.1038/s41562-020-0866-1

Competing interests

The author declares no competing interests. 